\title{
ARRAY DETECTORS AND INSTRUMENTS FOR THE ESO VLT
}

\author{
Sandro D'Odorico \\ European Southern Observatory
}

\begin{abstract}
Two-D array detectors are one of the key components of the instruments being built for the four ESO 8-m telescopes, the VLT project. Three optical and two infrared instruments are under construction. One faint object imager and multi-slit spectrograph to operate in the spectral region $350-1000 \mathrm{~nm}$ (acronym FORS) is based on a $2048^{2}$ highefficiency CCD. A two-channel echelle spectrograph to work in the $300-1000 \mathrm{~nm}$ region (UVRS) is built around a mosaic of $2048^{2}, 15 \mu \mathrm{m}$ CCDs. A multifiber, new spectrograph is based on a 4096 x 2048, $16 \mu \mathrm{m}$ CCD. The two infrared high-resolution instruments, one imager/long-slit spectrometer (ISAAC) and one high-resolution camera are designed to work with adaptive optics and in the speckle mode (CONICA). Both are designed to incorporate IR arrays up to a format of $1024^{2}$ and will work in the spectral region of $1-5 \mu \mathrm{m}$. The main properties of the instruments and of detectors are presented and their close interdependence is illustrated.
\end{abstract}

\section{THE VLT PROJECT OF ESO AND ITS INSTRUMENTATION}

The VLT project of the European Southern Observatory foresees the construction of four $8-\mathrm{m}$ telescopes to work between the atmospheric cutoff in the UV and $20 \mu \mathrm{m}$, complemented by an interferometric array of three $2-\mathrm{m}$ class telescopes. The project was funded at the end of 1987 and it is now being executed. The new observatory is being built on the Paranal Peak, a new astronomical site in the Atacama Desert in the north of Chile. First light at the first 8-m telescope is foreseen in 1998.

The VLT alt-azimuth 8-m unit telescope is described by Enard (1994) and its optics by Diericks (1994). In the first implementation every unit telescope will be equipped with two Nasmyth foci and a Cassegrain focus. At the time of this writing (November, 1994) there are six instruments under construction for the VLT. They are listed in Table 1 together with their main characteristics. More data can be found in the ESO publication "Instruments for the ESO VLT" edited by A. Moorwood (1994). There are another nine instruments under study for a possible implementation at the telescope (previous report and D'Odorico 1994). For the six approved first VLT instruments we plan to usee CCD detectors up to a format of $2048^{2}, 4096$ x 2048 and $4096^{2}$ for FORS, FUEGOS and UVES respectively and infrared arrays of 256 (current baseline) or $1024^{2}$ (possible upgrade) for ISAAC and CONICA.

\section{PROPERTIES OF MODERN CCD DETECTORS}

The paper by Finger et al. (1995) in these Proceedings presents an updated view of infrared arrays and I refer to it for a summary of their properties. In the following, I discuss the key properties of current CCD detectors with reference to the ESO experience in this area. 


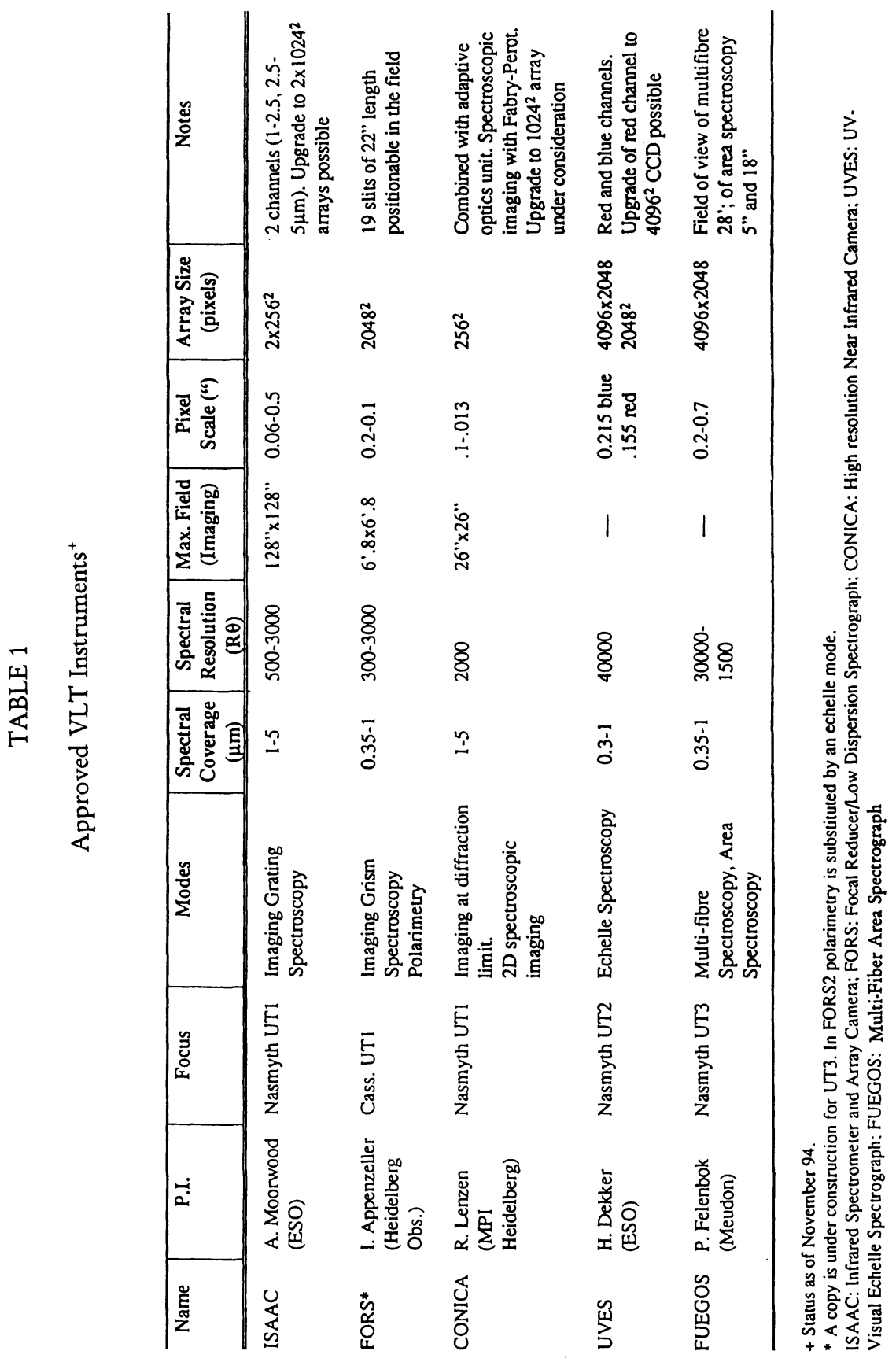




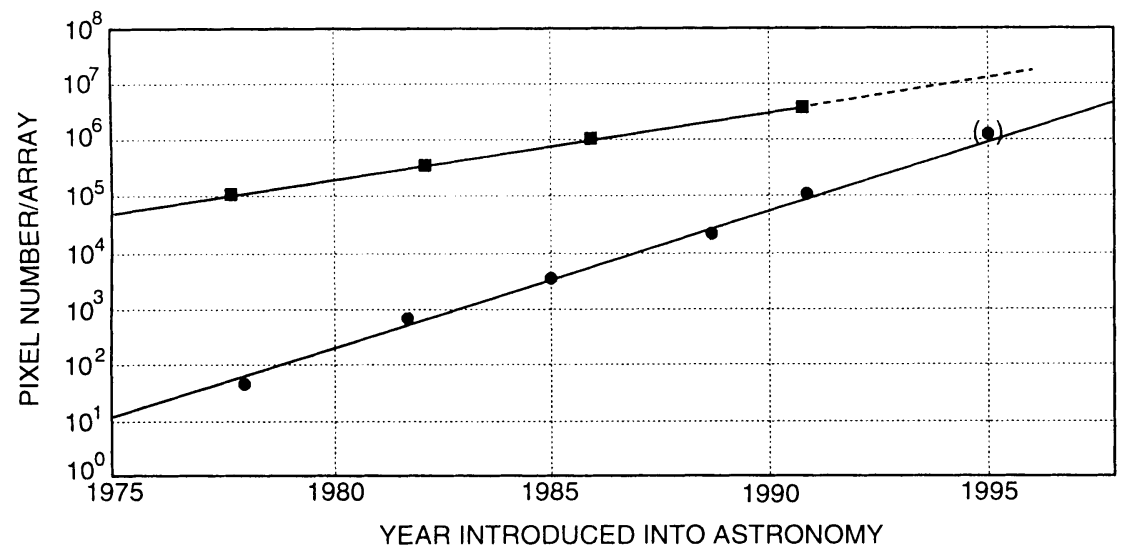

Fig. 1: Maximum number of pixels in array detectors at optical and infrared wavelengths (square and circles respectively) as a function of time. At optical wavelengths, no thinned device is routinely available at format larger than $2048^{2}$ although $4096 \times 2048$ format have been produced. At infrared wavelength, two manufacturers have announced $1024^{2}$ arrays for delivery in 1995.

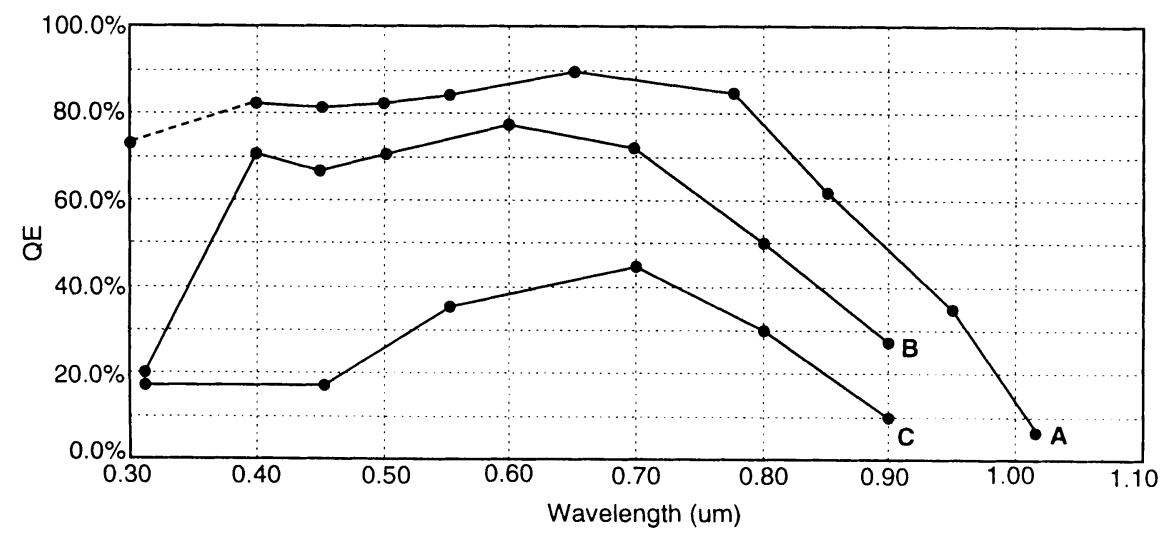

Fig. 2: Quantum efficiency curves of three ESO CCD devices. Curve A is a LORAL $2688 \mathrm{x}$ $512,15 \mu \mathrm{m}$ pixel CCD thinned for ESO by M. Lesser at the University of Arizona. The measurements have been taken after UV flooding. Curve B is a thinned $1024^{2}, 24 \mu \mathrm{m}$ SITe CCD. Curve $\mathrm{C}$ is a lumogen coated LORAL $2048^{2}, 15 \mu \mathrm{m}$ CCD. The last curve is indicative of the devices in use on the WF/PC 2 of the Hubble Space Telescope. 


\subsection{Pixel Size and Global Size}

Fig. 1 (on the previous page) shows how the maximum number of pixels/array has been steadily increasing for CCDs and for infrared arrays. For optical detectors the $2048^{2}$ format has become a standard product, but larger formats such as $4096^{2}$ and 4096 x 2048 are still in prototyping stage. The pixel size provided in the latest products is $15 \mu \mathrm{m}$, with the exception of the SITe $2048^{2}$ and $1024^{2}$ devices which have $24 \mu \mathrm{m}$ pixels. The choice of pixel size and CCD formats is dictated by the silicon technology which for most manufacturers is presently based on the use of four-inch wafers. Four 20482, $15 \mu \mathrm{m} \mathrm{CCD} \mathrm{devices} \mathrm{can} \mathrm{be} \mathrm{fitted} \mathrm{in} \mathrm{a} \mathrm{single}$ silicon wafer thus ensuring a relatively high yield to the process. When the scientific application requires a detector size larger than $30 \times 30 \mathrm{~mm}$, it is planned to achieve that by mosaicing of 2048 or $4096 \times 2048$ buttable CCDs.

For infrared arrays the change has been even more spectacular, with an increase in the number of pixels by four orders of magnitude in the last two decades.

\subsection{Quantum Efficiency}

As the telescopes are getting larger and the instruments more efficient it has become imperative that detectors operate at QEs close to 100\%. Fig. 2 (on the previous page) shows three typical QE curves for three CCDs used on ESO instruments. The high values neded for modern ground-based instruments can be obtained with thinned CCDs with a surface treatment and an antireflection coating only. Curve A, although obtained after flooding the cold CCD with UV light, sets a mark which should be the goal for future devices. If the CCD is warmed up, the high $\mathrm{QE}$ is reduced and the process has to be repeated. Future efforts should concentrate in obtaining a stable process for enhancing the sensitivity (Lesser 1994)

\subsection{Read-Out-Noise and Dark Current Noise}

While size and QE are possibly the two key properties of CCD detectors, read-out-noise and dark current play an important role in certain regimes of observations. At large telescopes this is the case for those observing modes where the detector noise dominates photon noise or sky backgound photon statistics, such as high resolution spectroscopy of faint objects. In this case the gain by going to a larger telescope is particularly relevant. For a given exposure time and limiting magnitude, the $\mathrm{S} / \mathrm{N}$ ratio to be obtained is proportional to $\mathrm{D}^{2}$, while it is proportional to $\mathrm{D}$ in the cases where the noise is dominated by photon statistics of the object or the sky.

With the improvement of on-chip electronics, the read-out-noise has considerably improved in the last two decades. Values of $30 \mathrm{e}^{-}$rms were the standard in 1980 while values $\leq 5 \mathrm{e}^{-}$are regularly achieved nowadays. This performance is measured at sampling rates of $20-50$ $\mu \mathrm{s} /$ pixel. More recently, those low values have been measured at faster rates as well (see Luppino et al. 1995 in these Proceedings).

The Poisson noise of thermally generated charge (dark current) is also significant in the 
observing modes which require very long integrations of faint sources. Again at large telescopes this is the case in high resolution spectroscopy. Dark current values can be reduced by operating the CCD at very low temperature but this often leads to a reduction of the QE. Dark current has been considerably reduced in modern CCDs when operated in Multi-Pinned-Phase mode (Janesick et al. 1989). At ESO we regularly achieve dark current values of less than 10 $2 \mathrm{re}^{-} /$hour at temperature of $-120^{\circ} \mathrm{C}$ with devices operated in MPP mode.

\subsection{Linearity, Full Well Capacity, Radiation Hits and Flatness}

In astronomical applications other CCD properties have to be taken into account carefully. Linearity over a broad dynamic range is usually quoted as one of the main advantages of CCD detectors with respect to both photographic plates and photon-counting detectors. Actually the manufacturers do not carry out any highly accurate tests of the linearity when a new device is produced. Linearity is also related to the CCD associated control electronics and it has to be verified eventually by the final user. Very few measurements on CCD linearity can be found in the literature. As these measurements require a stable laboratory setup and tedious testing, the ultimate deviations from a linear behavior in CCDs are poorly known. At ESO the linearity of the CCDs is measured at the time of characterization and later on monitored during the operation at the telescope, as described by Abbott (1995) in these proceedings. Fig. 3 shows the measured deviations from linearity for a SITe $1024^{2}$ thinned CCD. Apart from the measurements uncertainties at low level, the linearity is confirmed to better than $0.3 \%$ over four decades of recorded intensity values.

Radiation hits are charges produced by radioactive processes in the vicinity of the CCD, including the silicon itself or byproducts, typically muons, of interactions of cosmic particles with the Earth's atmosphere. The resulting charge can vary from a few electrons to a few hundreds and is normally concentrated on one to two pixels. There is no significant change in the event count rate when the CCD is moved from sea level to the observatory and the rates for a given CCD type are relatively constant. Out of $111024^{2}$ and $512^{2}$ thinned SITe CCDs received at ESO between 1990 and 1994, we obtain a radiation hit rate of $3.1 \pm 0.2$ events $/ \mathrm{cm}^{2} / \mathrm{min}$. At these rates the effect does degrade the signal to noise ratio of many types of observations and effectively limits the exposure time. On Loral unthinned CCDs we measure typically $1.3 \pm 0.1$ events in the same units, in agreement with the lower $\mathrm{QE}$ of these devices. A more dedicated effort should be made to pin down the possible local source of the contaminating radiation, which might well originate to a large fraction in the chip package or in the dewar materials.

A final property of CCDs which is very relevant to many astronomical applications is their flatness. Deviation from flatness may originate in the process of mounting the thinned CCDs on a substrate or in the poor quality of the substrate itself. When operated with fast optics these deviations can affect the final optical quality of the instrument. The specifications of future thinned CCDs call for deviations from flatness smaller than $\pm 15 \mu \mathrm{m}$. The current SITe $2048^{2}, 24 \mu \mathrm{m}$ thinned CCDs on the contrary show a difference of about $0.2 \mathrm{~mm}$ between the center and the corners of the device. This curved detector plane must be taken into account in the optical design of the instruments which use this type of detector. 
In view of the large number of VLT instruments which will rely on the use of CCDs, ESO has initiated a number of developments to secure high performance, standard detector systems to be operational on the VLT at first light. The main actions are reported in the next sections.

\subsection{Development of CCDs}

There are four VLT instruments under construction which require CCD detectors. For the two FORS instruments, ESO has already procured two SITe $2048^{2}$ thinned CCDs. The 24 $\mu \mathrm{m}$ pixels match well to the scale of the instrument giving a $0.20 \mathrm{arcsec} / \mathrm{pixel}$ and a total field of $6.8^{\prime} \times 6.8^{\prime}$. A change of collimator in the instrument provides a high resolution mode with a scale of $0.1 \mathrm{arcsec} /$ pixel over a smaller field. It is interesting to note that for these Focal Reducer/Spectrographs which will be installed at the Cassegrain foci of UT1 and UT3, there is no need for a CCD with more pixels. The field covered by the $2048^{2}$ SITe CCD is very close to the maximum field for an instrument at the Cassegrain focus. Some of the lenses in the focal reducer are also close to the maximum size which can be manufactured in the required FK54 glass material. The QE of the SITe CCDs (typically curve B in Fig. 2) is well matched to the transmission of this instrument which is high only above $400 \mathrm{~nm}$. The expected read-out-noise of $5 \mathrm{e}^{-}$typical for these chips is fully acceptable because the vast majority of observations with this instrument will be sky-background limited.

The other two VLT instruments UVES and FUEGOS are based on CCDs up to a format of $4096^{2}$ and $15 \mu \mathrm{m}$ pixels. High QE and the lowest possible read-out-noise are also required for the high resolution spectroscopy observations planned with these instruments. ESO is following two parallel routes for the procurement of the detectors for these instruments. Since 1992 we contracted the development of a $2048^{2}, 15 \mu \mathrm{m}$ thinned CCD, which can be butted on three sides, to Thomson CSF. The specifications for these CCDs (Iwert 1994) call for high performance devices which could be used to build up mosaics of two $\mathrm{x}$ two or larger. This new product identification is $7397 \mathrm{M}$. The delivery of five prototypes is currently planned for the first quarter of 1995. In parallel, ESO has obtained through a foundry effort with LORAL SOUTH (the former Ford Aerospace) a number of front side 20482, $15 \mu \mathrm{m}$ CCDs also buttable on three sides (original design by J. Geary and D. Bredthauer). ESO has contacted to M. Lesser of the Steward Observatory the thinning, packaging and antireflection treatment of the best CCDs of this lot. The first thinned devices are due for delivery at the end of 1994. The results obtained by Lesser on similar devices (see Fig. 2, curve A0), are encouraging. The CCDs will be mounted on the same package planned for the TH 7397 M CCDs.

For future VLT instruments ESO is considering participation in other development for the realization of high quality CCDs. There are two of them which appear well matched to our requirements for VLT instruments. Lincoln Lab at MIT is under discussion as a potential source of prototyping a $4096 \times 2048,15 \mu \mathrm{m}$ thinned device with high speed, low noise amplifiers, as reported by Luppino et al. (1995) at this meeting. The British company EEV is also entering into the development of a $4096 \times 2048,13.5 \mu \mathrm{m}$ thinned device for a consortium led by RGO. 


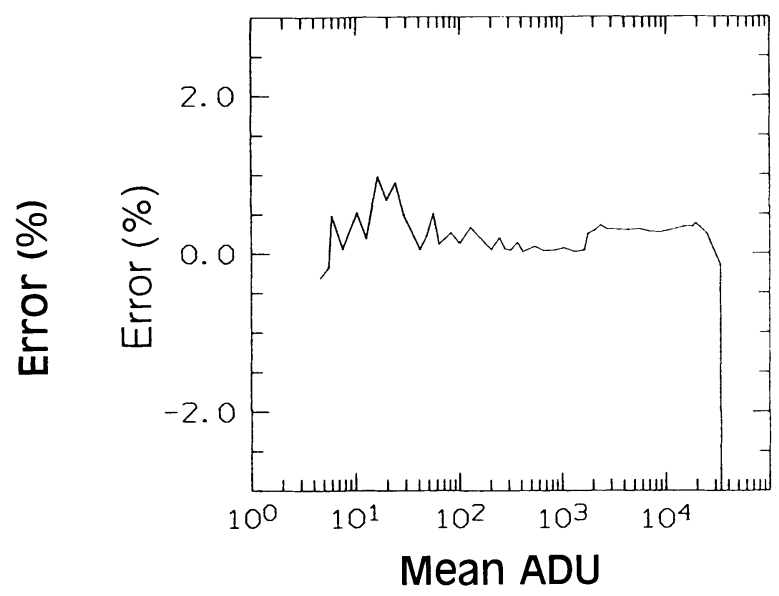

Fig. 3. Percentage deviations from the linearity for a SITe $1024^{2}$ thinned CCD recently tested at $\mathrm{ESO}$ as a function of intensity (One $\mathrm{ADU}=$ six $^{-}$). Noise below $200 \mathrm{ADU}$ is due to the uncertainties at low signal levels. The step at about $1200 \mathrm{ADU}$ is due to a calibration offset in the measuring device. Full well capacity of CCDs (defined as the charge value at which the deviation from linearity exceeds $1 \%$ )is related to the pixel size and to the mode of operation. With read-out-noise lower than five $\mathrm{e}^{-}$and 16 bit ADCs it is possible to work at full resolution with full well capacities up to $150,000-200,000 \mathrm{e}^{-}$. These values are normally achieved with $24 \mu \mathrm{m}$ CCDs. Devices operated in MPP and with smaller pixel size are in our experience limited to less than $100,000 \mathrm{e}^{-}$.

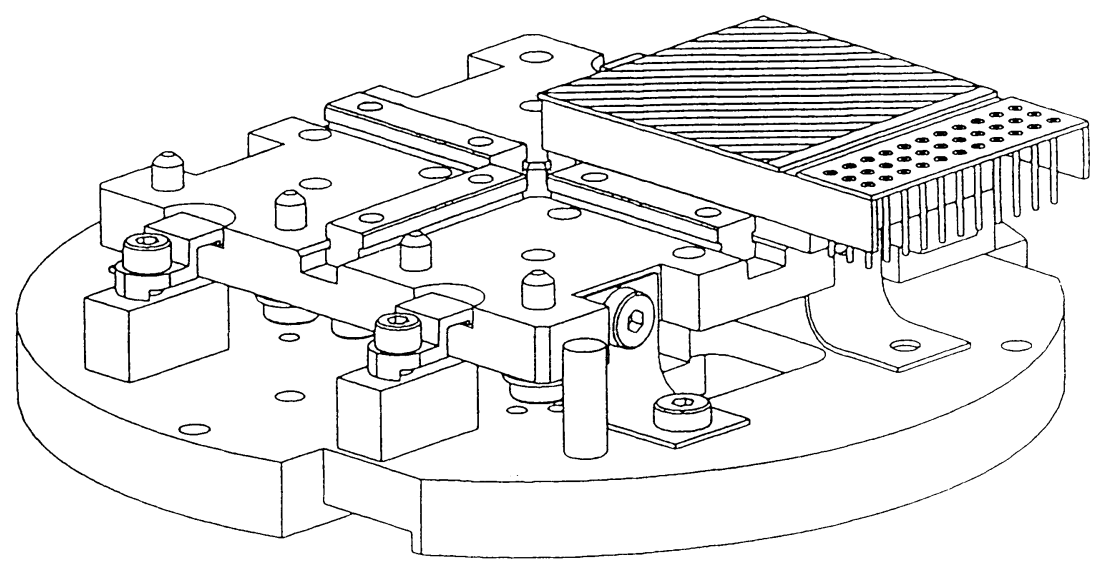

Fig. 4: A CAD view of part of the new ESO detector head in the configuration prepared for a mosaic of two $x$ two large, buttable CCDs. One of the packaged devices is also shown in place. The design foresees the building up of a mosaic giving an overall flatness of better that $30 \mu \mathrm{m}$ peak-to-peak with a minimum of adjustment required for the CCD alignement. 


\subsection{The New ESO Detector Controller ACE}

A new CCD controller based on the use of transputer modules and DSPs has been developed at ESO to be used with single large size CCD or with mosaics of them as foreseen for the VLT instruments. The new controller, ACE, is described by Reiss (1994). The design has been driven by the need to handle with speed and low system noise modern large size detectors. The controller had also to be compact and to produce less than 25 watts. In parallel to controller development, ESO has designed a new CCD mounting head and associated cryogenics. The new standard head is designed to be simpler to integrate than the current one, with the possibility of fine adjustment of tilt and position of the detector surface while still keeping the very high stability which is required for long integration. It can accomodate mosaics of up to two $\mathrm{x}$ two $2048^{2} \mathrm{CCDs}$ for a total of 16 outputs. The detector front head in the mosaic case is shown in the CAD picture reproduced in Fig. 4.

\section{REFERENCES}

Abbott, T. 1995 in IAU Symposium No.167, New Developments in Array Technology and Applications, A. G. D. Philip, K. A. Janes and A. R. Upgren, eds., Kluwer Academic Pub., Dordrecht, p. 343

Diericks, P. 1994 ESO VLT Technical Report 526

D'Odorico, S. 1994 ESO Scientific Report No.15

Enard, D. 1994 Proc SPIE 2199, 394

Finger, G., Nicolini, G., Biereichal, P., Meyer, M. and Moorwood, A. F. M. 1995 in IAU Symposium No. 167, New Developments in Array Technology and Applications, A. G. D. Philip, K. A. Janes and A. R. Upgren, eds., Kluwer Academic Pub., Dordrecht, p. 81

Iwert, O. 1994 VLT-ESO 13600-0268

Janesick, J., Elliott, T., Fraschetti G., Collins, S., Blouke, M. and Corrie B. 1989 Proc. SPIE 1071, 15

Lesser, M. P. 1994 Proc. SPIE 2198, 782

Luppino, G. A., Metzger, M. R. and Miyazaki, S. 1995 in IAU Symposium No. 167, New Developments in Array Technology and Applications, A. G. D. Philip, K. A. Janes and A. R. Upgren, eds., Kluwer Academic Pub., Dordrecht, p. 297

Moorwood, A., ed. 1994 Instruments for the ESO VLT, ESO, Garching

Reiss, R. 1994 Proc. SPIE 2198, 895 


\section{DISCUSSION}

PHILIP: The VLT will operate first as four separate telescopes but there are plans to operate it as one telescope. When will this be done?

D'ODORICO: The layout of the observatory includes light ducts which lead from the unit telescopes to a central laboratory. Both coherent light addition (interferometry) and incoherent combination are foreseen. At present this part of the program will be implemented starting in 2000. Preparatory studies are continuing.

COHEN: Are you building one of each instrument or four of them?

D'ODORICO: While the intial instrument plan foresaw a large amount of duplication, at present one instrument only, the Focal Reducer Spectrograph, is being built in two eventually identical copies which will go to telescopes one and three.

COHEN: Is ESO spending money on CCD development? If so, how much and with what companies? You have spoken only of commerically available CCDs.

D'ODORICO: In the past two years, ESO has spent on average half a million DM/year in CCD development with industry or resource laboratories. For the details, please refer to the paper by Iwert. 